\title{
Martingale Densities for General Asset Prices
}

\author{
Martin Schweizer \\ Universität Bonn \\ Institut für Angewandte Mathematik \\ Wegelerstraße 6 \\ D-W-5300 Bonn 1 \\ Germany
}

\begin{abstract}
This paper discusses some properties of general asset prices in continuous time. We introduce the concept of a martingale density which is a generalization of an equivalent martingale measure, and we show that absence of arbitrage plus some technical conditions implies the existence of a martingale density. This is in turn already sufficient to derive a recent result of Back (1990) on local risk premia for asset returns. As an application, we obtain a simple condition, valid in arbitrary information structures, for the drift part of discounted security gains to be absolutely continuous with respect to the variance process of the martingale part.
\end{abstract}

(Journal of Mathematical Economics 21 (1992), 363-378)

Financial support by Deutsche Forschungsgemeinschaft, Sonderforschungsbereich 303 at the University of Bonn, is gratefully acknowledged. 


\section{Introduction}

Most papers on continuous-time finance rely on two basic assumptions: asset price processes are continuous (or even Itô processes), and there exists an equivalent martingale measure or risk-neutral probability measure which determines security prices as expected discounted pay-outs. The purpose of this paper is to explore the extent to which one can relax these assumptions while still preserving nice properties of asset prices. An important step in this direction was recently taken by Back (1990). He showed that one can allow security gains to be special semimartingales and still obtain a CAPM-like formula for local risk premia if one has an equivalent martingale measure. In particular, continuity of asset prices can be dispensed with. We generalize Back's result by introducing the notion of a martingale density. Given a security gains process $G$, a martingale density for $G$ is a local martingale $Z$ with $Z_{0}=1$ such that the product of $Z$ and the discounted gains process $\widetilde{G}$ is a local martingale. In the special case where $Z$ is a strictly positive martingale and the product is a true martingale, $Z$ obviously determines an equivalent martingale measure. We show that the existence of a strictly positive martingale density is already sufficient to obtain Back's theorem, and we also establish a converse result. Furthermore, we derive results on the structure of asset prices satisfying a mild integrability condition and admitting a strict martingale density.

The paper is structured as follows. In section 1, we describe the basic model and introduce the concept of a martingale density. Using results of Ansel/Stricker (1991), we show that a suitable formulation of absence of arbitrage implies that security gains must be special semimartingales, a property which was simply assumed by Back (1990). Under some additional nondegeneracy and integrability assumptions, we then prove that a martingale density $\widehat{Z}$ for $G$ exists. In fact, we can even give a fairly explicit formula for $\widehat{Z}$ in terms of quantities related to the semimartingale decomposition of $G$.

Section 2 then contains applications of martingale densities. Throughout that section, we suppose that the gains process is a special semimartingale, the justification being provided by the results in section 1. First we derive Back's formula for local risk premia from the assumption that $G$ admits a strictly positive martingale density. By systematically using Yoeurp's lemma, we can also give a simplification of the original proof and establish a converse result. This shows in particular that Back's formula can be interpreted as a characterization of martingale densities. As an application, we prove that existence of a strictly positive martingale density plus a weak integrability condition implies that asset prices must have a certain structure: the drift part of the discounted gains process must be absolutely continuous with respect to the variance process of the martingale part. For a Brownian filtration, this means that discounted gains must be Itô processes if there exists 
a strictly positive martingale density. This is a generalization of results of Huang (1985a, 1985b) who worked with an equivalent martingale measure. Finally, we give a characterization of martingale densities $Z$ in terms of an equation which $Z$ must satisfy. Under the same nondegeneracy and integrability assumptions used in section 1, we furthermore provide a parametrization of all locally square-integrable martingale densities by the set of all locally square-integrable local martingales $R$ which are orthogonal to $\widetilde{M}$, the martingale part of the discounted gains process $\widetilde{G}$. This extends previous results of several authors who only considered the case of a Brownian filtration.

\section{Existence of martingale densities}

We start essentially from the same basic model for securities as in Back (1990). This model was introduced by Harrison/Pliska (1981) and extended by Huang (1985b) to include interim cash payments. We refer to Back (1990) for the underlying motivation and to Dellacherie/Meyer (1982), hereafter abbreviated as D/M, for precise definitions of the probabilistic concepts. The uncertainty is described by a probability space $(\Omega, \mathcal{F}, P)$ with a filtration $\mathbb{F}=\left(\mathcal{F}_{t}\right)_{0 \leq t \leq T}$ satisfying the usual conditions of right-continuity and completeness. $T>0$ denotes a fixed time horizon. There are $d+1$ securities. One of these, denoted by $Y=\left(Y_{t}\right)_{0 \leq t \leq T}$, is assumed to be locally riskless in the sense that it has continuous paths of finite variation. Furthermore, $Y$ is strictly positive and normalized to have $Y_{0}=1$. The remaining $d$ risky securities are described by their ex-dividend price processes $S^{i}=\left(S_{t}^{i}\right)_{0 \leq t \leq T}$ and their cumulative cash pay-outs $D^{i}=\left(D_{t}^{i}\right)_{0 \leq t \leq T}$. We assume that both $S$ and $D$ are adapted processes with right-continuous trajectories having left limits. The corresponding gains process is then $G=S+D$ and the discounted gains process $\widetilde{G}$ is given by

$$
\widetilde{G}_{t}=\frac{S_{t}}{Y_{t}}+\int_{0}^{t} \frac{1}{Y_{u}} d D_{u} \quad, \quad 0 \leq t \leq T .
$$

We remark that the second term in (1.1) is defined by partial integration as

$$
\int_{0}^{t} \frac{1}{Y_{u}} d D_{u}:=\frac{D_{t}}{Y_{t}}-D_{0}+\int_{0}^{t} \frac{D_{u-}}{Y_{u}^{2}} d Y_{u}
$$

and thus requires no additional assumptions on $D$. Note also that (1.1) and (1.2) imply that $G$ is a (special) semimartingale if and only if $\widetilde{G}$ is a (special) semimartingale. 
It is well known that the absence of arbitrage opportunities is essentially equivalent to the existence of an equivalent martingale measure $Q$ for $\widetilde{G}$. We say "essentially" because this statement depends on the precise definition of an arbitrage opportunity; see Kreps (1981), Back/Pliska (1991), Dalang/Morton/Willinger (1990), Stricker (1990) for more detailed discussions of this problem. An equivalent martingale measure $Q$ for $\widetilde{G}$ is a probability measure which is equivalent to $P$ (i.e., $Q$ and $P$ have the same null sets), satisfies $Q=P$ on $\mathcal{F}_{0}$ and has the property that $\widetilde{G}$ is a martingale with respect to $Q$. The equivalence of $Q$ and $P$ implies that the Radon-Nikodym derivative $Z_{T}:=\frac{d Q}{d P}$ exists and defines a strictly positive martingale $Z$ with $Z_{0}=1$ by

$$
Z_{t}:=E\left[Z_{T} \mid \mathcal{F}_{t}\right]=\left.\frac{d Q}{d P}\right|_{\mathcal{F}_{t}} \quad, \quad 0 \leq t \leq T .
$$

The martingale property of $\widetilde{G}$ under $Q$ is then equivalent to saying that the product $\widetilde{G} Z$ is a $P$-martingale. The process $\varrho=\frac{Z}{Y}$ is called a state price density. This discussion motivates the following

Definition. A local $P$-martingale $Z$ with $Z_{0}=1$ is called a martingale density for $\widetilde{G}$ if the process $\widetilde{G} Z$ is a local $P$-martingale. $Z$ is called a strict martingale density if, in addition, $Z$ is strictly positive. In both cases, the process $\varrho:=\frac{Z}{Y}$ is called a generalized state price density.

Remarks. 1) Being a nonnegative local martingale, a strict martingale density $Z$ is always a supermartingale. It is a (uniformly integrable) martingale if and only if $E\left[Z_{T}\right]=1$. There are quite general results on sufficient conditions for this (see Jacod (1979), chapter VIII-2), but these conditions are often rather restrictive or hard to verify. Furthermore, there are quite natural situations where one has a strict martingale density, but no equivalent martingale measure. For instance, Cheng (1991) and Elliott/Kopp (1991) study this problem for Brownian bridge processes. Another class of examples is provided by models with an infinite time horizon; see Foldes (1989).

2) Let $Z$ be a strict martingale density and set $d Q=Z_{T} d P$. Then $Q$ is obviously a measure with the same null sets as $P$. In general, however, $Q$ is only a sub-probability, i.e., $Q[\Omega] \leq 1 ; Q$ is a probability if and only if $Z$ is a uniformly integrable martingale. But many properties with respect to $Q$ can be reformulated in terms of $Z$ and $P$ alone, and these formulations still make sense for $Q[\Omega]<1$. For instance, we have just seen that the (local) martingale property of $\widetilde{G}$ under $Q$ corresponds to $\widetilde{G} Z$ being a (local) $P$-martingale. We shall illustrate in section 2 how the use of martingale densities instead of martingale measures leads to very general and yet quite elegant formulations.

3) Notice that in contrast to Back (1990), we do not assume so far that $G$ (or $S$, or $D$ ) is a semimartingale. We shall see in Proposition 1 that this property 
follows for $G$ from a no-arbitrage condition. For $S$ and $D$, however, no specific assumptions (except adaptedness and right-continuous trajectories with left limits) will be required in the paper. It is also worth pointing out that the existence of a strict martingale density $Z$ for $G$ implies that $G$ is a semimartingale. In fact, this follows immediately from the fact that $\widetilde{G} Z$ is a local martingale and $\frac{1}{Z}$ is a semimartingale.

Our goal is now to show that the absence of arbitrage opportunities, combined with a technical condition on $G$, implies the existence of a martingale density $Z$ for $G$. To make this more precise, we need some notation. Following Ansel/Stricker (1991), we define an elementary predictable process $H=\left(H_{t}\right)_{0 \leq t \leq T}$ to be a process of the form

$$
H_{t}=\sum_{i=0}^{n-1} \xi_{i} I_{\left(t_{i}, t_{i+1}\right]}(t)
$$

for some $n \in \mathbb{N}$, where $0 \leq t_{0}<t_{1}<\ldots<t_{n} \leq T$ and each $\xi_{i}$ is an $\mathbb{R}^{d_{-}}$ valued $\mathcal{F}_{t_{i}}$-measurable random variable. Intuitively, $H$ corresponds to a trading strategy which consists of holding the portfolio $\xi_{i}$ during the time interval $\left(t_{i}, t_{i+1}\right]$. The discounted trading gains generated by this strategy are then given by the "elementary stochastic integral"

$$
(H \cdot \widetilde{G})_{T}:=\sum_{i=0}^{n-1}\left(\xi_{i}, \widetilde{G}_{t_{i+1}}-\widetilde{G}_{t_{i}}\right)
$$

where we write $(\cdot, \cdot)$ for the scalar product in $\mathbb{R}^{d}$. We denote by $\mathcal{K}$ the set of all random variables $(H \cdot \widetilde{G})_{T}$ where $H$ runs over all bounded elementary predictable processes and by $\overline{\mathcal{K}}$ the closure of $\mathcal{K}$ in $\mathcal{L}^{1}(P)$. Finally, $\mathcal{L}_{+}^{1}(P)$ is the set of all nonnegative integrable random variables on $(\Omega, \mathcal{F}, P)$.

Definition. We say that $G$ admits no arbitrage opportunities if

$$
\overline{\mathcal{K}} \cap \mathcal{L}_{+}^{1}(P)=\{0\} .
$$

This condition was introduced by Stricker (1990) who proved the following result: If $\widetilde{G}$ is a continuous adapted $\mathbb{R}^{d}$-valued process with $\widetilde{G}_{t} \in \mathcal{L}^{p}(P)$ for all $t \in[0, T]$ and some $p \in[1, \infty)$, then the property

$$
\overline{\mathcal{K}}^{p} \cap \mathcal{L}_{+}^{p}(P)=\{0\}
$$

(with ${ }^{-p}$ denoting closure in $\mathcal{L}^{p}(P)$ ) is equivalent to the existence of an equivalent martingale measure $Q$ for $\widetilde{G}$ with $\frac{d Q}{d P} \in \mathcal{L}^{q}(P)$, where $\frac{1}{p}+\frac{1}{q}=1$. The case $p=\infty$ was recently solved by Delbaen (1991). For general (not necessarily continuous) asset prices, we have the following result by Ansel/Stricker (1991). 
Proposition 1. Suppose that $G$ admits no arbitrage opportunities and that $\sup _{0 \leq t \leq T}\left|\widetilde{G}_{t}\right| \in \mathcal{L}^{2}(P)$. Then $\widetilde{G}$ is a special semimartingale with canonical decompo$0 \leq t \leq T$

sition

$$
\widetilde{G}=\widetilde{G}_{0}+\widetilde{M}+\widetilde{A}
$$

where for each component $i=1, \ldots, d, \widetilde{M}^{i}$ is a locally square-integrable local martingale under $P$ with $\widetilde{M}_{0}^{i}=0$, and

$$
\widetilde{A}_{t}^{i}=\int_{0}^{t} \widetilde{\alpha}_{u}^{i} d\left\langle\widetilde{M}^{i}\right\rangle_{u}^{P} \quad, \quad 0 \leq t \leq T
$$

for a predictable process $\widetilde{\alpha}^{i}$ satisfying

$$
\int_{0}^{T}\left(\widetilde{\alpha}_{u}^{i}\right)^{2} d\left\langle\widetilde{M}^{i}\right\rangle_{u}^{P}<\infty \quad P-\text { a.s. }
$$

(As a matter of fact, Ansel/Stricker (1991) state and prove this theorem for $d=1$, but their proof still works for $d>1$ if one argues separately for each component.)

We can use the preceding result to give an existence theorem for martingale densities. Under the assumptions of Proposition 1, set

$$
\begin{aligned}
B_{t} & :=\sum_{i=1}^{d}\left\langle\widetilde{M}^{i}\right\rangle_{t}^{P} \quad, \quad 0 \leq t \leq T, \\
\sigma_{t}^{i j} & :=\frac{d\left\langle\widetilde{M}^{i}, \widetilde{M}^{j}\right\rangle_{t}^{P}}{d B_{t}} \quad, \quad 0 \leq t \leq T, \quad i, j=1, \ldots, d, \\
\gamma_{t}^{i} & :=\widetilde{\alpha}_{t}^{i} \sigma_{t}^{i i} \quad, \quad 0 \leq t \leq T, \quad i=1, \ldots, d .
\end{aligned}
$$

Let ${ }^{*}$ denote transposition, $\mathcal{L}_{\text {loc }}^{2}(\widetilde{M})$ the set of all predictable $\mathbb{R}^{d}$-valued processes $\lambda$ such that $\int \lambda_{u}^{*} \sigma_{u} \lambda_{u} d B_{u}$ is locally integrable, $\mathcal{M}_{\mathrm{loc}, 0}^{2}(P)$ the set of all locally square-integrable local martingales under $P$ starting from 0 , and $\Delta \widetilde{M}_{t}:=\widetilde{M}_{t}-\widetilde{M}_{t-}$ the jump of $\widetilde{M}$ in $t \in[0, T]$. For every $\lambda \in \mathcal{L}_{\text {loc }}^{2}(\widetilde{M})$, the stochastic integral $\int \lambda_{u} d \widetilde{M}_{u}$ is well-defined and in $\mathcal{M}_{\text {loc }, 0}^{2}(P)$; see Jacod (1979), chapter IV-2. Finally, the stochastic exponential $\mathcal{E}(X)$ of a semimartingale $X$ is the solution $U$ of the stochastic differential equation

$$
U_{t}=1+\int_{0}^{t} U_{s-} d X_{s}
$$


Theorem 2. Suppose that $G$ admits no arbitrage opportunities and that $\sup _{0 \leq t \leq T}\left|\widetilde{G}_{t}\right| \in \mathcal{L}^{2}(P)$. Suppose also that

the matrix $\sigma_{t}(\omega)$ is invertible for $P \times B$-a.e. $(\omega, t) \in \Omega \times[0, T]$

and

$$
\text { the process } \widehat{\lambda}_{t}:=\sigma_{t}^{-1} \gamma_{t}, 0 \leq t \leq T \text {, is in } \mathcal{L}_{\text {loc }}^{2}(\widetilde{M}) .
$$

Then $\widehat{Z}:=\mathcal{E}\left(-\int \widehat{\lambda} d \widetilde{M}\right)$ is a martingale density for $G$. $\widehat{Z}$ is a strict martingale density if and only if

$$
\widehat{\lambda}_{t} \Delta \widetilde{M}_{t}<1 \quad P-\text { a.s. for every } t \in[0, T] .
$$

Proof. Since $\widehat{Z}$ is locally square-integrable by (1.7), the first assertion follows immediately from Proposition 5. The second is a direct consequence of the properties of the stochastic exponential; see Protter (1990).

q.e.d.

Remark. Intuitively, the nondegeneracy condition (1.6) means that there are no redundant assets. The idea for such a condition is taken from Karatzas/Lehoczky/ Shreve/Xu (1991).

\section{Applications}

In this section, we give some applications of martingale densities. First of all, we generalize a theorem of Back (1990) on local risk premia. Then we show that under a weak integrability condition, the existence of a martingale density implies that asset prices must have a certain structure. This can be used to generalize results of Huang (1985a, 1985b). Finally we provide a characterization and a parametrization of martingale densities.

Throughout this section, we shall assume that the gains process $G=S+D$ is a special semimartingale with canonical decomposition

$$
G=G_{0}+M+A,
$$

where the local martingale $M$ and the predictable finite-variation process $A$ both have initial value 0 . This assumption can be justified by Proposition 1. By (1.2) and Itô's lemma, the discounted gains process $\widetilde{G}$ is then also a special semimartingale whose canonical decomposition $\widetilde{G}=S_{0}+\widetilde{M}+\widetilde{A}$ is given by

$$
\begin{aligned}
\widetilde{M}_{t} & =\int_{0}^{t} \frac{1}{Y_{u}} d M_{u}, \\
\widetilde{A}_{t} & =\int_{0}^{t} \frac{1}{Y_{u}} d A_{u}-\int_{0}^{t} \frac{S_{u-}}{Y_{u}^{2}} d Y_{u} .
\end{aligned}
$$


We recall from Back (1990) the definition of the covariance process of two special semimartingales $U=U_{0}+M^{U}+A^{U}$ and $V=V_{0}+N^{V}+B^{V}$ : If the product $M^{U} M^{V}$ is a special semimartingale, then

$$
\langle U, V\rangle_{t}:=U_{0} V_{0}+\left\langle M^{U}, M^{V}\right\rangle_{t}^{P}+\sum_{0 \leq s \leq t} \Delta A_{s}^{U} \Delta A_{s}^{V}
$$

where $\left\langle M^{U}, M^{V}\right\rangle^{P}$ is the usual sharp bracket process from martingale theory (D/M, VII.39) and $\Delta A_{s}^{U}=A_{s}^{U}-A_{s-}^{U}$ is the jump of $A^{U}$ at time $s$.

Theorem 3. a) Let $Z$ be a strict martingale density for $G$ and $\varrho$ the corresponding generalized state price density. If $M \varrho$ is a special semimartingale, then

$$
\int_{0}^{t} \frac{d A_{u}^{i}}{S_{u-}^{i}}-\int_{0}^{t} \frac{d Y_{u}}{Y_{u}}=-\int_{0}^{t} \frac{d\left\langle G^{i}, \varrho\right\rangle_{u}}{S_{u-}^{i} \varrho_{u-}} \quad P-\text { a.s. }
$$

for all $t \in[0, T]$ and $i=1, \ldots, d$.

b) Conversely, let $Z$ be a local $P$-martingale with $Z_{0}=1$ and $\varrho=\frac{Z}{Y}$. If (2.4) holds, then $M \varrho$ is a special semimartingale and $Z$ is a martingale density for $G$.

Proof. 1) Let us first prove the direct half a). We start by noting that $\left[Z, \widetilde{A}^{i}\right]$ is a local $P$-martingale by Yoeurp's lemma (D/M, VII.36). Thus, the product rule (D/M, VIII.18)

$$
d\left(Z \widetilde{A}^{i}\right)=Z_{-} d \widetilde{A}^{i}+\widetilde{A}_{-}^{i} d Z+d\left[Z, \widetilde{A}^{i}\right]
$$

shows that $Z \widetilde{A}^{i}$ is a special semimartingale. Since $Z$ is a martingale density, this implies that $Z \widetilde{M}^{i}=Z \widetilde{G}^{i}-Z S_{0}^{i}-Z \widetilde{A}^{i}$ is also special so that $\left\langle Z, \widetilde{M}^{i}\right\rangle^{P}$ exists. Now we apply the product rule and (2.2) to obtain

$$
\begin{aligned}
d\left(\widetilde{G}^{i} Z\right)= & \widetilde{G}_{-}^{i} d Z+Z_{-} d \widetilde{G}^{i}+d\left[Z, \widetilde{G}^{i}\right] \\
= & \widetilde{G}_{-}^{i} d Z+Z_{-} d \widetilde{M}^{i}+d\left[Z, \widetilde{A}^{i}\right]+d\left[Z, \widetilde{M}^{i}\right]-d\left\langle Z, \widetilde{M}^{i}\right\rangle^{P} \\
& +\frac{Z_{-}}{Y} d A^{i}-\frac{Z_{-} S_{-}^{i}}{Y^{2}} d Y+d\left\langle Z, \widetilde{M}^{i}\right\rangle^{P} \\
= & \text { local P-martingale }+\left(\frac{Z_{-}}{Y} d A^{i}-\frac{Z_{-} S_{-}^{i}}{Y^{2}} d Y+d\left\langle Z, \widetilde{M}^{i}\right\rangle^{P}\right),
\end{aligned}
$$

again using Yoeurp's lemma. But since $\widetilde{G}^{i} Z$ is also a local $P$-martingale, the last term, being predictable and of finite variation, must vanish identically. This shows that we always have

$$
\frac{d A^{i}}{S_{-}^{i}}-\frac{d Y}{Y}=-\frac{Y d\left\langle Z, \widetilde{M}^{i}\right\rangle^{P}}{S_{-}^{i} Z_{-}}=-\frac{d\left\langle Z, \widetilde{M}^{i}\right\rangle^{P}}{S_{-}^{i} \varrho_{-}} .
$$


2) To conclude the proof of a), we now identify the numerator on the righthand side of (2.5). Since $M^{i} \varrho$ is special by assumption, so is $M^{i} Z=M^{i} \varrho Y$ by the product rule. Thus $\left\langle M^{i}, Z\right\rangle^{P}$ exists. Furthermore,

$$
d \varrho=d\left(\frac{Z}{Y}\right)=\frac{1}{Y} d Z-\frac{Z_{-}}{Y^{2}} d Y
$$

yields by (2.3) and the continuity of $Y$

$$
\begin{aligned}
d\left\langle G^{i}, \varrho\right\rangle & =d\left\langle M^{i}, \int \frac{1}{Y} d Z\right\rangle^{P} \\
& =\frac{1}{Y} d\left\langle M^{i}, Z\right\rangle^{P} \\
& =d\left\langle\int \frac{1}{Y} d M^{i}, Z\right\rangle^{P} \\
& =d\left\langle\widetilde{M}^{i}, Z\right\rangle^{P},
\end{aligned}
$$

and combining this with (2.5) proves (2.4).

3) To prove the converse half b), we use the product rule, (2.2), (2.4) and Yoeurp's lemma to obtain

$$
\begin{aligned}
d\left(\widetilde{G}^{i} Z\right) & =\widetilde{G}_{-}^{i} d Z+Z_{-} d \widetilde{M}^{i}+\frac{Z_{-}}{Y} d A^{i}-\frac{Z_{-} S_{-}^{i}}{Y^{2}} d Y+d\left[Z, \widetilde{M}^{i}\right]+d\left[Z, \widetilde{A}^{i}\right] \\
& =\text { local } P \text {-martingale }+d\left[Z, \widetilde{M}^{i}\right]+\frac{Z_{-} S_{-}^{i}}{Y} \cdot\left(\frac{d A^{i}}{S_{-}^{i}}-\frac{d Y}{Y}\right) \\
& =\text { local } P \text {-martingale }+d\left[Z, \widetilde{M}^{i}\right]-d\left\langle G^{i}, \varrho\right\rangle .
\end{aligned}
$$

If we can show that $M^{i} \varrho$ is special, part 2) of the proof will imply that $\left\langle Z, \widetilde{M}^{i}\right\rangle^{P}$ exists and equals $\left\langle G^{i}, \varrho\right\rangle$, thus proving $\widetilde{G}^{i} Z$ to be a local $P$-martingale. But the validity of (2.4) implies in particular the existence of $\left\langle G^{i}, \varrho\right\rangle$, and therefore $M^{i} \cdot \int \frac{1}{Y} d Z$ must be special by (2.3). Thus

$$
M^{i} \varrho=M^{i} \cdot \frac{Z}{Y}=M^{i} \cdot \int \frac{1}{Y} d Z-M^{i} \cdot \int \frac{Z_{-}}{Y^{2}} d Y
$$

is also special.

q.e.d.

Remarks. 1) The direct half of Theorem 3 generalizes a result of Back (1990) who proved (2.4) assuming the existence of an equivalent martingale measure. Working instead with martingale densities allows us to avoid additional integrability conditions. Furthermore, the systematic use of Yoeurp's lemma has led to a simplification of the original proof. 
2) Back's interpretation of (2.4) is that it describes the local risk premium $\frac{d A_{t}^{i}}{S_{t-}^{i}}-\frac{d Y_{t}}{Y_{t}}$ of a security as the negative covariance of that security's relative price change with the relative change in the state price density, $-\frac{d\left\langle G^{i}, \varrho\right\rangle_{t}}{S_{t-}^{i} \varrho_{t-}}$. Combining a) and b) of Theorem 3, we see that (2.4) is also essentially equivalent to the local martingale property of $\widetilde{G} Z$ under $P$ and thus can be interpreted as an alternative characterization of martingale densities.

As an application of Theorem 3, we deduce the following structure result for asset prices:

Proposition 4. Suppose that $Z$ is a strict martingale density for $G$ and that $M$ and $Z$ are both locally square-integrable. Then each $\widetilde{A}^{i}$ is absolutely continuous with respect to $\left\langle\widetilde{M}^{i}\right\rangle^{P}$, i.e.,

$$
\widetilde{A}_{t}^{i}=\int_{0}^{t} \widetilde{\alpha}_{u}^{i} d\left\langle\widetilde{M}^{i}\right\rangle_{u}^{P} \quad, \quad 0 \leq t \leq T
$$

for some predictable process $\widetilde{\alpha}^{i} \in \mathcal{L}_{\text {loc }}^{2}\left(P \times\left\langle\widetilde{M}^{i}\right\rangle^{P}\right)$.

Proof. Combining (2.2) and (2.5) yields

$$
d \widetilde{A}^{i}=\frac{S_{-}^{i}}{Y} \cdot\left(\frac{d A^{i}}{S_{-}^{i}}-\frac{d Y}{Y}\right)=-\frac{d\left\langle Z, \widetilde{M}^{i}\right\rangle^{P}}{Z_{-}}
$$

But since $Z$ and $\widetilde{M}^{i}=\int \frac{1}{Y} d M^{i}$ are both locally square-integrable, the KunitaWatanabe projection theorem (D/M, VIII.52) implies that

$$
d\left\langle Z, \widetilde{M}^{i}\right\rangle^{P}=\zeta^{i} d\left\langle\widetilde{M}^{i}\right\rangle^{P}
$$

for some predictable process $\zeta^{i} \in \mathcal{L}_{\text {loc }}^{2}\left(P \times\left\langle\widetilde{M}^{i}\right\rangle^{P}\right)$. Since $\frac{1}{Z_{-}}$is locally bounded, (2.6) clearly holds with $\widetilde{\alpha}^{i}=-\frac{\zeta^{i}}{Z_{-}}$.

q.e.d.

Remark. Proposition 4 can be viewed as a sort of converse to Theorem 2 where we used the absolute continuity of $\widetilde{A}^{i}$ with respect to $\left\langle\widetilde{M}^{i}\right\rangle^{P}$ to construct a martingale density. Note also that the basic assumption is the local square-integrability of $M^{i}$ (or equivalently $\widetilde{M}^{i}$ ). This condition is indispensable for the existence of $\left\langle M^{i}\right\rangle^{P}$ or $\left\langle\widetilde{M}^{i}\right\rangle^{P}$; see Yoeurp (1976). In view of the results of Stricker (1990) mentioned in section 1 , local square-integrability of $Z$ is then a quite natural assumption. In 
particular, these conditions are always satisfied if both $M$ and $Z$ are continuous or have locally bounded jumps.

Example. Suppose that the filtration $\mathbb{F}$ is generated by an $n$-dimensional Brownian motion $\left(\beta^{1}, \ldots, \beta^{n}\right)$. Then it is well known that every local martingale of $\mathbb{F}$ is a sum of stochastic integrals with respect to $\beta^{1}, \ldots, \beta^{n}$ and in particular continuous, hence locally bounded. If we now assume the existence of a strict martingale density $Z$ (which is weaker than the existence of an equivalent martingale measure $Q)$, Proposition 4 implies that $\widetilde{A}^{i} \ll\left\langle\widetilde{M}^{i}\right\rangle^{P}$. But since $\widetilde{M}^{i}$ is a sum of stochastic integrals of Wiener processes, $\left\langle\widetilde{M}^{i}\right\rangle^{P}$ is absolutely continuous. Hence we deduce that the discounted gains process $\widetilde{G}$ must be an Itô process of the form

$$
d \widetilde{G}_{t}^{i}=\sum_{j=1}^{n} \vartheta_{t}^{i j} d \beta_{t}^{j}+\mu_{t}^{i} d t \quad, \quad i=1, \ldots, d
$$

for some predictable processes $\vartheta^{i j}$ and $\mu^{i}$. Thus, we are led in a very natural way to the standard diffusion model used in this situation. In particular, the existence of a strict martingale density $Z$ rules out the rather nasty possibility that the drift terms $\widetilde{A}^{i}$ could be continuous processes of finite variation which are not absolutely continuous. See Harrison/Pitbladdo/Schaefer (1984) for an illustrative example of such a process.

Remarks. 1) This example generalizes results of Huang (1985a, 1985b) who assumed the existence of an equivalent martingale measure. It should be pointed out here that the preceding structure results depend crucially on the absence of consumption over time. For a detailed study of how agents' preferences over temporal consumption patterns affect the behaviour of asset prices in the absence of arbitrage opportunities, we refer to Hindy/Huang (1989).

2) Note that absolute continuity of $\widetilde{A}$ does not necessarily imply absolute continuity of $A$. In fact, since

$$
A_{t}=\int_{0}^{t} Y_{u} d \widetilde{A}_{u}+\int_{0}^{t} \frac{S_{u-}}{Y_{u}} d Y_{u}
$$

by (2.2), this implication is only true if the riskless asset $Y$ is itself absolutely continuous. See Karatzas/Lehoczky/Shreve (1990) for an example of a situation where discounted gains are Itô processes, but gains themselves are not.

So far, we have discussed some implications of the existence of a strict martingale density. Now every equivalent martingale measure $Q$ for $\widetilde{G}$ gives rise to a strict martingale density $Z$, and it is well known that there is a unique equivalent martingale measure if and only if we have a complete model; see Harrison/Pliska 
(1981, 1983) and Jarrow/Madan (1990). It is therefore of interest to characterize martingale densities in more detail, and this is the objective of the next result.

Proposition 5. Suppose that $\widetilde{G}=S_{0}+\widetilde{M}+\widetilde{A}$ is a special semimartingale with $\widetilde{M} \in \mathcal{M}_{\text {loc }, 0}^{2}(P)$ and

$$
\widetilde{A}_{t}^{i}=\int_{0}^{t} \widetilde{\alpha}_{u}^{i} d\left\langle\widetilde{M}^{i}\right\rangle_{u}^{P} \quad, \quad 0 \leq t \leq T, \quad i=1, \ldots, d
$$

with $\widetilde{\alpha}^{i} \in \mathcal{L}_{\text {loc }}^{2}\left(P \times\left\langle\widetilde{M}^{i}\right\rangle^{P}\right)$ for each $i$.

a) $Z$ is a martingale density for $G$ if and only if for each $i=1, \ldots, d$, there exists a local martingale $N^{i}$ with $N_{0}^{i}=0$ and orthogonal to $\widetilde{M}^{i}$ (i.e., $N^{i} \widetilde{M}^{i}$ is a local martingale) such that $Z$ satisfies the equation

$$
Z_{t}=1-\int_{0}^{t} Z_{u-} \widetilde{\alpha}_{u}^{i} d \widetilde{M}_{u}^{i}+N_{t}^{i} \quad P-a . s .
$$

for all $t \in[0, T]$.

b) Suppose in addition that (1.6) and (1.7) are satisfied. Then $Z$ is a martingale density and locally square-integrable if and only if $Z$ satisfies

$$
Z_{t}=1-\int_{0}^{t} Z_{u-} \widehat{\lambda}_{u} d \widetilde{M}_{u}+R_{t} \quad P-a . s .
$$

on $[0, T]$ for some $R \in \mathcal{M}_{\text {loc }, 0}^{2}(P)$ orthogonal to $\widetilde{M}$.

Proof. 1) Let $Z$ be any local martingale with $Z_{0}=1$ and define the local martingales $N^{i}(i=1, \ldots, d)$ by

$$
N_{t}^{i}:=Z_{t}-1+\int_{0}^{t} Z_{u-} \widetilde{\alpha}_{u}^{i} d \widetilde{M}_{u}^{i} \quad, \quad 0 \leq t \leq T .
$$

To prove a), we have to show that $\widetilde{G}^{i} Z$ is a local martingale if and only if $N^{i} \widetilde{M}^{i}$ is a local martingale. To see that this is true, we use successively the product rule, Yoeurp's lemma, (2.7), the fact that $\int Z_{-} \widetilde{\alpha}^{i} d\left[\widetilde{M}^{i}\right]-\int Z_{-} \widetilde{\alpha}^{i} d\left\langle\widetilde{M}^{i}\right\rangle^{P}$ is a local martingale, the definition of $N^{i}$ and the fact that $N^{i} \widetilde{M}^{i}-\left[N^{i}, \widetilde{M^{i}}\right]$ is a local 
martingale to obtain

$$
\begin{aligned}
d\left(\widetilde{G}^{i} Z\right) & =\widetilde{G}_{-}^{i} d Z+Z_{-} d \widetilde{G}^{i}+d\left[Z, \widetilde{G}^{i}\right] \\
& =\text { local } P \text {-martingale }+Z_{-} d \widetilde{A}^{i}+d\left[Z, \widetilde{M}^{i}\right] \\
& =\text { local } P \text {-martingale }+Z_{-} \widetilde{\alpha}^{i} d\left\langle\widetilde{M}^{i}\right\rangle^{P}+d\left[Z, \widetilde{M}^{i}\right] \\
& =\text { local } P \text {-martingale }+Z_{-} \widetilde{\alpha}^{i} d\left[\widetilde{M}^{i}\right]+d\left[Z, \widetilde{M}^{i}\right] \\
& =\text { local } P \text {-martingale }+d\left[N^{i}, \widetilde{M}^{i}\right] \\
& =\text { local } P \text {-martingale }+d\left(N^{i} \widetilde{M}^{i}\right) .
\end{aligned}
$$

2) Suppose that $Z$ is a martingale density and locally square-integrable. By the Kunita-Watanabe projection theorem, $Z$ can be written as

$$
Z_{t}=1+\int_{0}^{t} \zeta_{u} d \widetilde{M}_{u}+R_{t}^{0} \quad, \quad 0 \leq t \leq T
$$

with $\zeta \in \mathcal{L}_{\text {loc }}^{2}(\widetilde{M})$ and $R^{0} \in \mathcal{M}_{\text {loc }, 0}^{2}(P)$ orthogonal to $\widetilde{M}$. Now define the processes $\vartheta:=\zeta+Z_{-} \widehat{\lambda} \in \mathcal{L}_{\text {loc }}^{2}(\widetilde{M})$ and $R \in \mathcal{M}_{\text {loc }, 0}^{2}(P)$ by

$$
R_{t}:=R_{t}^{0}+\int_{0}^{t} \vartheta_{u} d \widetilde{M}_{u} \quad, \quad 0 \leq t \leq T .
$$

Since $Z$ then clearly satisfies $(2.9)$ and $R^{0}$ is orthogonal to $\widetilde{M}$, it only remains to show that $\int \vartheta d \widetilde{M}$ is orthogonal to $\widetilde{M}$. To see this, we first compute

$$
\begin{aligned}
\left\langle\widetilde{M^{i}}, \int \vartheta d \widetilde{M}\right\rangle^{P} & =\sum_{j=1}^{d} \int_{0}^{t} \vartheta_{u}^{j} d\left\langle\widetilde{M}^{i}, \widetilde{M}^{j}\right\rangle_{u}^{P} \\
& =\int_{0}^{t} \sum_{j=1}^{d} \sigma_{u}^{i j} \vartheta_{u}^{j} d B_{u} \\
& =\int_{0}^{t}(\sigma \vartheta)_{u}^{i} d B_{u} .
\end{aligned}
$$

Thus we have to show that

$$
\sigma \vartheta=0 \quad P \times B \text {-a.e. on } \Omega \times[0, T]
$$

or equivalently

$$
\sigma \zeta=-Z_{-} \gamma \quad P \times B \text {-a.e. on } \Omega \times[0, T] \text {. }
$$


But since $Z$ is a martingale density, successively applying the definition of $\gamma$, the definition of $\sigma,(2.8),(2.10)$ and again the definition of $\sigma$ yields

$$
\begin{aligned}
-\int_{0}^{t} Z_{u-} \gamma_{u}^{i} d B_{u} & =-\int_{0}^{t} Z_{u-} \widetilde{\alpha}_{u}^{i} \sigma_{u}^{i i} d B_{u} \\
& =-\int_{0}^{t} Z_{u-} \widetilde{\alpha}_{u}^{i} d\left\langle\widetilde{M^{i}}, \widetilde{M}^{i}\right\rangle_{u}^{P} \\
& =\left\langle Z, \widetilde{M}^{i}\right\rangle_{t}^{P} \\
& =\sum_{j=1}^{d} \int_{0}^{t} \zeta_{u}^{j} d\left\langle\widetilde{M}^{j}, \widetilde{M}^{i}\right\rangle_{u}^{P} \\
& =\int_{0}^{t} \sum_{j=1}^{d} \zeta_{u}^{j} \sigma_{u}^{j i} d B_{u} \\
& =\int_{0}^{t}(\sigma \zeta)_{u}^{i} d B_{u} P-\text { a.s. }
\end{aligned}
$$

on $[0, T]$ and therefore (2.11).

3) Conversely, suppose that $Z$ satisfies (2.9). If we define $N^{i} \in \mathcal{M}_{\mathrm{loc}, 0}^{2}(P)$ by

$$
N_{t}^{i}:=R_{t}+\int_{0}^{t} Z_{u-} \widetilde{\alpha}_{u}^{i} d \widetilde{M}_{u}^{i}-\int_{0}^{t} Z_{u-} \widehat{\lambda}_{u} d \widetilde{M}_{u}
$$

we obviously have (2.8) and

$$
\begin{aligned}
\left\langle N^{i}, \widetilde{M}^{i}\right\rangle_{t}^{P} & =\left\langle R, \widetilde{M}^{i}\right\rangle_{t}^{P}+\int_{0}^{t} Z_{u-} \widetilde{\alpha}_{u}^{i} d\left\langle\widetilde{M}^{i}\right\rangle_{u}^{P}-\sum_{j=1}^{d} \int_{0}^{t} Z_{u-} \widehat{\lambda}_{u}^{j} d\left\langle\widetilde{M}^{j}, \widetilde{M}^{i}\right\rangle_{u}^{P} \\
& =\int_{0}^{t} Z_{u-}\left(\gamma_{u}^{i}-(\sigma \widehat{\lambda})_{u}^{i}\right) d B_{u}=0
\end{aligned}
$$

by (1.7), so $N^{i}$ is orthogonal to $\widetilde{M}^{i}$.

q.e.d.

Remarks. 1) The idea for the proof of a) is taken from Ansel/Stricker (1991); a previous version of this paper treated only the locally square-integrable case. Note that equation (2.9) allows us to parametrize the set of all locally square-integrable 
martingale densities by the space $\mathcal{R}$ of all local martingales $R \in \mathcal{M}_{\text {loc }, 0}^{2}(P)$ which are orthogonal to $\widetilde{M}$. For a Brownian filtration, results similar to b) have also been obtained by He/Pearson (1990), Karatzas/Lehoczky/Shreve/Xu (1991) and El Karoui/Quenez (1991).

2) A slightly more general form of equation (2.9) has been studied by Yoeurp/ Yor (1977). In particular, these authors show that (2.9) has a unique solution $Z$ (whose explicit expression is rather complicated) for given $\widehat{\lambda}, \widetilde{M}$ and $R$. Since the above reference is not easily accessible, we mention that this result is also proved in chapter VI-1 of Jacod (1979).

The simplest case of equation (2.9) is of course the case $R \equiv 0$. The equation then reduces to

$$
Z_{t}=1-\int_{0}^{t} Z_{u-} \widehat{\lambda}_{u} d \widetilde{M}_{u} \quad, \quad 0 \leq t \leq T
$$

whose solution $\widehat{Z}$ is given by the stochastic exponential $\mathcal{E}\left(-\int \widetilde{\lambda} d \widetilde{M}\right)$ which has already appeared in Theorem 2. We shall call $\widehat{Z}$ the minimal martingale density for $G$ since it is in a sense the simplest martingale density: If we think of $G$ and $Y$ as given, we also know the decomposition $\widetilde{G}=S_{0}+\widetilde{M}+\widetilde{A}$ and the processes $\widetilde{\alpha}^{i}$ and $\sigma^{i j}$ appearing in (2.7) and (1.7), respectively. But clearly this is already sufficient to construct $\widehat{Z}$. Put differently, the exact knowledge of the second order structure of $\widetilde{G}$ is enough to determine $\widehat{Z}$.

If $\widehat{Z}$ is not only a martingale density, but even a strictly positive squareintegrable martingale, $d \widehat{P}=\widehat{Z}_{T} d P$ defines an equivalent martingale measure $\widehat{P}$ for $\widetilde{G}$ which is called the minimal equivalent martingale measure. $\widehat{P}$ has the property that it preserves martingales: if $L$ is a square-integrable $P$-martingale $P$ orthogonal to $\widetilde{M}$, then $L$ is also a $\widehat{P}$-martingale. Thus, $\widehat{P}$ is minimal among all equivalent martingale measures for $\widetilde{G}$ in the sense that it turns $\widetilde{G}$ into a martingale, but preserves the remaining structure of the model. More details on these questions can be found in Föllmer/Schweizer (1991); Schweizer (1991a, 1991b) gives applications to the problem of hedging contingent claims. Finally we mention that for the case of a Brownian filtration, a similar concept was also studied by Pagès (1989) and He/Pearson (1990).

Acknowledgments. I am grateful to the referees whose helpful comments and suggestions have resulted in a major revision of the paper and have thus led to improvements in several points. 


\section{References}

J.-P. Ansel and C. Stricker (1991), "Lois de Martingale, Densités et Décomposition de Föllmer-Schweizer", preprint, Université de Franche-Comté, Besançon

K. Back (1990), "Asset Pricing for General Processes”, preprint, to appear in Journal of Mathematical Economics

K. Back and S. R. Pliska (1991), "On the Fundamental Theorem of Asset Pricing with an Infinite State Space", Journal of Mathematical Economics 20, $1-18$

S. Cheng (1991), "On the Feasibility of Arbitrage-Based Option Pricing when Stochastic Bond Price Processes are Involved", Journal of Economic Theory 53, $185-198$

R. C. Dalang, A. Morton and W. Willinger (1990), "Equivalent Martingale Measures and No-Arbitrage in Stochastic Securities Market Models", Stochastics and Stochastics Reports 29, 185-201

F. Delbaen (1991), "Representing Martingale Measures when Asset Prices are Continuous and Bounded", preprint, Vrije Universiteit Brussel

C. Dellacherie and P.-A. Meyer (1982), "Probabilities and Potential B", North-Holland

N. El Karoui and M.-C. Quenez (1991), "Programmation Dynamique et Evaluation des Actifs Contingents en Marché Incomplet", preprint, Université Paris VI

R. J. Elliott and P. E. Kopp (1991), "Equivalent Martingale Measures for Bridge Processes", preprint, University of Alberta, Edmonton

L. Foldes (1989), "Conditions for Optimality in the Infinite-Horizon Portfoliocum-Saving Problem with Semimartingale Investments", working paper, London School of Economics

H. Föllmer and M. Schweizer (1991), "Hedging of Contingent Claims under Incomplete Information", in: "Applied Stochastic Analysis", eds. M. H. A. Davis and R. J. Elliott, Stochastics Monographs vol. 5, Gordon and Breach, London/New York, 389-414

J. M. Harrison, R. Pitbladdo and S. M. Schaefer (1984), "Continuous Price Processes in Frictionless Markets Have Infinite Variation", Journal of Business $57 / 3,353-365$

J. M. Harrison and S. R. Pliska (1981), "Martingales and Stochastic Integrals in the Theory of Continuous Trading", Stochastic Processes and their Applications 
$11,215-260$

J. M. Harrison and S. R. Pliska (1983), "A Stochastic Calculus Model of Continuous Trading: Complete Markets", Stochastic Processes and their Applications $15,313-316$

H. He and N. D. Pearson (1990), "Consumption and Portfolio Policies with Incomplete Markets and Short-Sale Constraints: The Infinite Dimensional Case", preprint, Haas School of Business, University of California at Berkeley

A. Hindy and Chi-fu Huang (1989), "On Intertemporal Preferences with a Continuous Time Dimension II: The Case of Uncertainty", preprint, Sloan School of Management, MIT

Chi-fu Huang (1985a), "Information Structure and Equilibrium Asset Prices", Journal of Economic Theory 35, 33-71

Chi-fu Huang (1985b), "Information Structures and Viable Price Systems", Journal of Mathematical Economics 14, 215-240

J. Jacod (1979), "Calcul Stochastique et Problèmes de Martingales", Lecture Notes in Mathematics 714, Springer

R. A. Jarrow and D. B. Madan (1990), "A Characterization of Complete Security Markets on a Brownian Filtration", preprint, Johnson Graduate School of Management, Cornell University

I. Karatzas, J. P. Lehoczky and S. E. Shreve (1990), "Equilibrium Models with Singular Asset Prices", research report No. 90-95, Carnegie Mellon University

I. Karatzas, J. P. Lehoczky, S. E. Shreve and G.-L. Xu (1991), "Martingale and Duality Methods for Utility Maximization in an Incomplete Market", SIAM Journal on Control and Optimization 29, 702-730

D. M. Kreps (1981), "Arbitrage and Equilibrium in Economies with Infinitely Many Commodities", Journal of Mathematical Economics 8, 15-35

H. Pagès (1989), "Three Essays in Optimal Consumption", Ph.D. thesis, MIT

P. Protter (1990), "Stochastic Integration and Differential Equations. A New Approach", Springer

M. Schweizer (1991a), "Option Hedging for Semimartingales", Stochastic Processes and their Applications 37, 339-363

M. Schweizer (1991b), "Mean-Variance Hedging for General Claims", Annals of Applied Probability (to appear)

C. Stricker (1990), "Arbitrage et Lois de Martingale", Annales de l'Institut 
Henri Poincaré 26, 451-460

Ch. Yoeurp (1976), "Décompositions des Martingales Locales et Formules Exponentielles", Séminaire de Probabilités X, Lecture Notes in Mathematics 511, Springer, 432-480

Ch. Yoeurp and M. Yor (1977), "Espace Orthogonal à une Semi-Martingale et Applications", unpublished 